\title{
Don't Kill the Suspense: How Outcome Knowledge Influences the Enjoyment of Entertainment (an Abstract)
}

\author{
Mujde Yuksel, Elizabeth Miller, and Easwar Iyer
}

\begin{abstract}
Technological advances have enabled consumers to take greater control over how and when they view television programming, with many consumers choosing to time-shift consumption. Yet, preferences for live viewing vs. time-shifting viewing vary across program types, with news, special event, and sports programming showing higher real-time viewing than other program types. While indeterminacy is one factor that has been shown to predict consumer preferences for real-time broadcasts, drawing on literature related to the uncertainty of outcome and suspense, we argue that there is more to this preference than indeterminacy alone. More specifically, we propose that entertainment experiences contain two types of suspense (outcome and process suspense) and we show how process suspense impacts consumer preferences for time-shifting in TV entertainment consumption. Our research enriches our understanding of the nature of suspense and the relationship between outcome knowledge and the ability to experience suspense while also providing practical insights into why consumers choose to time-shift some programs, but not others.
\end{abstract}

\footnotetext{
M. Yuksel $(\bowtie)$

Suffolk University, Boston, MA, USA

e-mail: myuksel@suffolk.edu

E. Miller • E. Iyer

University of Massachusetts Amherst, Amherst, MA, USA

e-mail: emiller@isenberg.umass.edu; iyer@isenberg.umass.edu 